\title{
Evaluación de competencias profesionales en la formación universitaria de ingenierías en informática
}

Carlos Gustavo López. Universidad de Buenos Aires | José Manuel Cabo Hernández y José Miguel Vílchez González. Universidad de Granada

Recepción: 21 de septiembre de 2015 | Revisión: 28 de septiembre de 2015 | Aceptación/Publicación: 5 de octubre de 2015 Correspondencia: glopez@fi.uba.ar

Citar: López, CG., Cabo Hernández, JM. y Vílchez González, JM. (2015). Evaluación de competencias profesionales en la formación universitaria de ingenierías en informática. ReiDoCrea, 4, 260-276. [http://hdl.handle.net/10481/38089]

\begin{abstract}
Resumen: Un modelo universitario de enseñanza aprendizaje basado en competencias supone un reto que implica no solo una innovación metodológica en la práctica docente sino también tecnológica, por la utilización de entornos virtuales de aprendizaje diseñados de forma específica para el desarrollo de competencias a partir de tareas contextualizadas acordes con el perfil profesional que se está formando y, finalmente, de modificaciones en las herramientas y técnicas de evaluación. Se presenta en este artículo, en relación a la innovación en evaluación, el proceso de elaboración y validación por expertos de un instrumento de evaluación de competencias basado en el uso de rúbricas, a aplicar en formación universitaria de Ingeniería en Informática en Argentina. El diseño parte de la definición de las competencias y pasa por la validación de la rúbrica por parte de 10 expertos. Se ofrece como conclusión la rúbrica generada.
\end{abstract}

Palabras clave: Evaluación | Competencias del docente

Evaluation of generic skills training in university computer engineering

Abstrac: A university teaching-learning model based on competences is a challenge that involves not only a methodological innovation in teaching but also technological, for the use of virtual learning environments designed specifically for skills development from contextualized tasks chords with the professional profile being formed and eventually changes in the tools and techniques of evaluation. The development and validation process is presented in this article, in relation to innovation in assessment by experts of a skills assessment tool based on the use of rubrics, to apply to university education in Computer Engineering in Argentina. The design part of the definition of powers and go through the validation of the heading by 10 experts. Heading generated is offered in conclusion.

Keywords: Evaluation | Teacher qualifications

Esta investigación se inició en el marco del Proyecto de Investigación UBATIC "Innovación Pedagógica vía TIC para la mejora de la calidad educativa en la FIUBA" (UBATIC 2011-2014). Resolución de Consejo Superior 3833/11. La investigación continúa en el Proyecto de Investigación UBATIC sobre "Diseño y Elaboración de material estandarizado para el desarrollo de habilidades sociales de comunicación" (UBATIC 2015-2016). Ambos financiados por la Secretaría de Asuntos Académicos de la Universidad de Buenos Aires y dirigidos por Gustavo López.

\section{Introducción}

Los denominados modelos basados en competencias no son una novedad reciente. En el ámbito laboral ya se recurría a ellos en los años 70 del siglo pasado, y a finales de los 80 suponían una apuesta de futuro.

La creación del Espacio Europeo de Educación Superior, a partir del año 2000, extiende los modelos basados en competencias a las titulaciones universitarias, con su reflejo en todo el mundo incluyendo el Espacio Latinoamericano de Educación Superior. Así, la Recomendación del Parlamento Europeo y del Consejo sobre las competencias clave para el aprendizaje permanente, de 18 de diciembre de 2006 (2006/962/CE), señala definitivamente como "marco de referencia" los sistemas basados en el desarrollo de competencias para toda la vida (Medina Rivilla, 2013).

El proyecto Tunning Latinoamérica (Beneitone y col., 2007) constata la extensión del modelo basado en competencias al continente americano tras los pasos de la Unión Europea y la OCDE; ha sido suficiente gestionar las evaluaciones externas desde el ámbito internacional para que los sistemas de enseñanza nacionales hayan confluido 
hacia un proceso de reformas curriculares basadas en un modelo de enseñanza aprendizaje basado en competencias.

Queda para otro momento valorar si las reformas curriculares en las etapas universitarias y no universitarias responden a las necesidades de las personas o de las empresas que tienen que emplear a las personas, porque en el contexto de la educación superior no podemos dejar de desarrollar competencias profesionales, precisamente por el ajuste cada vez mayor de la enseñanza aprendizaje a la "empleabilidad", por lo que estamos ajustando el currículo universitario a lo que las empresas demandan de sus trabajadores.

En el presente trabajo se ha tomado a las competencias genéricas como aquellas capacidades básicas, para la carrera de Ingeniería en Informática, que permiten desarrollar las competencias profesionales a partir de ellas.

No creemos necesario documentar la idea de que los sistemas de enseñanza son resistentes al cambio, y la de que toda reforma curricular implica un proceso de transformación; entre las presiones externas que los sistemas universitarios nacionales estarían recibiendo en este momento podemos destacar las siguientes (Salinas, 2004):

- Los cambios derivados del EEES y/o ELES.

- Los cambios propiciados por las TIC.

- Los cambios en el conocimiento.

- Los cambios en el ciudadano, en las necesidades de una persona formada, como persona "competente" en lo personal y en lo profesional.

El modelo de enseñanza aprendizaje basado en competencias puede entenderse como una alternativa a la situación actual. Rodríguez (2008) analiza los cambios derivados de sustituir un modelo basado en objetivos por otro basado en competencias, que ha de comenzar por el cambio metodológico implícito de un modelo que ha de centrarse en los estudiantes y en el diseño de situaciones de aprendizaje contextualizadas, complejas, dirigidas al desarrollo de capacidades, para su aplicación en la resolución de problemas, lo que a su vez provoca cambios en los sistemas de evaluación.

Pero no solo se trata de cubrir las innovaciones docentes que las competencias plantean para el diseño de las tareas y la evaluación de las mismas, sino que también hay que considerar innovador el diseño de las herramientas de seguimiento y evaluación del proceso continuo de desarrollo de las competencias (Neira y col., 2014).

Ligado a la sociedad del conocimiento y la información, es fácil referirse a la utilización de las TIC en las enseñanzas universitarias y su vinculación con el cambio docente implícito en cualquier reforma educativa. Este hecho se destaca tanto en el conjunto de reformas del sistema obligatorio como en la incorporación del crédito en el sistema universitario internacional como sistema de medida intercambiable del esfuerzo del estudiante, al menos en el Espacio Europeo de Educación Superior y en el Espacio Latinoamericano de Educación Superior.

Un modelo de aprendizaje basado en competencias lleva igualmente a la necesidad de contemplar diferentes contextos de las competencias profesionales que se intentan desarrollar desde las asignaturas universitarias, lo que, desde el aula, implica su consideración en ambientes virtuales de aprendizaje mediante, por ejemplo, 
simulaciones. En consecuencia, debe existir un cierto grado de especificidad de las herramientas tecnológicas, pues las simulaciones están muy ligadas a contextos muy precisos como puede ser, por ejemplo, las simulaciones clínicas en ámbitos de la salud, muy diferentes a las de las ingenierías.

En conclusión, un modelo universitario de enseñanza aprendizaje basado en competencias supone un reto que implica no solo una innovación metodológica sino también la utilización de entornos virtuales de aprendizaje diseñados de forma específica para el desarrollo de competencias, a partir de tareas contextualizadas acordes con el perfil profesional que se está formando. Entornos de aprendizaje que deben permitir, además, la evaluación del desarrollo de estas competencias.

\section{Entornos virtuales de aprendizaje}

No cabe duda de que uno de los hitos más importantes de la historia de la informática es la aparición de la World Wide Web, de la mano de Tim Berners-Lee, hacia 1989. Internet evolucionó y pasó a convertirse en el cyber-espacio (Berners-Lee 2002), palabra cuyo primer término deriva de cybernetics (del griego, kybernētēs), que significa "piloto". Fue utilizada por primera vez en la novela de William Gibson "Neuromante" (1984), y en la actualidad se utiliza, según la Real Academia Española de la Lengua, para describir al "ámbito artificial creado por medios informáticos". El cyber-espacio se ha ido transformando y ha llegado a constituir una extensión de la realidad física hasta llegar a ser considerado como un "lugar" en el cual pueden llevarse a cabo una cantidad innumerable de actividades humanas.

Los currículos actuales admiten la necesidad de aprovechar las facilidades brindadas por las TIC a los fines de la enseñanza, principalmente debido a las indudables ventajas didácticas que se han ido poniendo de manifiesto en diferentes trabajos de investigación. El software educativo posee características que resultan interesantes, tales como el acceso a todo tipo de información, la posibilidad de simular fenómenos y situaciones difíciles de observar en la realidad, la interactividad con el usuario, o la posibilidad de llevar a cabo un proceso de aprendizaje y evaluación individualizada (Pontes, 2005).

Según Pontes (2005) las ventajas que presentan las TIC en relación con las tres categorías de contenidos a cubrir en cualquier proceso de enseñanza-aprendizaje son:

- Contenidos conceptuales. Facilitan el acceso a la información e influyen en el aprendizaje de conceptos.

- Contenidos procedimentales. El manejo de Internet estimula el desarrollo de destrezas intelectuales como la capacidad indagadora, el autoaprendizaje o la familiarización con el uso de las TIC.

- Contenidos actitudinales. El uso de programas interactivos y la búsqueda de información en Internet ayuda a fomentar la actividad de los alumnos durante el proceso educativo, favoreciendo el intercambio de ideas, la motivación y el interés por su aprendizaje.

Una verdadera revolución digital no sucede únicamente por la apropiación tecnológica; también requiere nuevos enfoques de trabajo, para lo que es necesario partir de una racionalidad tecnicista en cuanto a los modos de conocer y de aprender. Surge de este modo un proceso de adaptación que supone cambios en los modelos educativos, entre ellos la modificación de los escenarios en los que ocurre el aprendizaje que, en general, se denominan "entornos virtuales de aprendizaje" (EVA). Los EVA son aplicaciones informáticas que ayudan en el aprendizaje asistido por computadoras (e- 
learning), cuya influencia en el proceso educativo varía en función del alcance y profundidad en la interacción e inmersión que se les brinde (Hung, 2002).

En consonancia con las tendencias constructivistas del aprendizaje, en las que el estudiante interactúa con todo su entorno desenvolviéndose en el medio y teniendo práctica en el contexto circundante (Sánchez, 2007), es de suma importancia tener en cuenta estas características durante el diseño de un EVA. Además, un EVA debe contemplar información detallada sobre el curso y datos para registro del estudiante.

Un tipo de EVA que destaca por su variada funcionalidad es la simulación, que proporciona una representación dinámica del funcionamiento de un sistema (Raviolo, 2010). Esto le ha proporcionado cada vez mayor relevancia en la enseñanza de un gran número de disciplinas científico-tecnológicas, puesto que permite visualizar el desarrollo de procesos mostrando la evolución del sistema representado y la interacción entre los elementos que lo integran o, al menos, algunas consecuencias de tales interacciones (Pontes, 2005). En estos ambientes, también denominadas laboratorios interactivos de simulación o laboratorios virtuales, se modelizan contextos reales en los que el estudiante debe participar de forma activa, característica por la que se pueden considerar estrategias de aprendizaje que pueden promover el desarrollo de competencias. Tienen aplicaciones en la enseñanza cuando se utilizan para la presentación de situaciones no asequibles en la práctica o que pueden ser peligrosas, o la de aquellas que requieren un equipo muy complejo (Pontes, 2005). Algunos de ellos incluyen módulos de contenidos teóricos sobre los fenómenos que se representan y permiten realizar actividades de aprendizaje, como la resolución de problemas relacionados con tales fenómenos.

El desarrollo de aplicaciones integrales de simulación constituye un proceso de mayor complejidad respecto de las soluciones convencionales, y requiere para su tratamiento del aporte de especialistas de diferentes áreas de conocimiento. De esta forma, dicho proceso se cimienta sobre la base de tres ejes de desarrollo, complementarios y dependientes entre sí (Hung, 2002):

- Educativo o Instruccional. Definición de las necesidades educativas primordiales a cubrir por la aplicación.

- Comunicacional. Define la interfaz del usuario (zona de interacción usuarioaplicativo). La usabilidad y accesibilidad del sistema, aplicada a entornos altamente interactivos, constituyen factores fundamentales en este apartado.

- Soporte Tecnológico. Adecuación de las posibilidades tecnológicas de desarrollo, integrando los ejes anteriores y validando la completitud del modelo global diseñado mediante la comprobación de la satisfacción de los requerimientos globales detectados.

En concordancia con las actuales estrategias de enseñanza aprendizaje, en las que se asume que lo esencial no es proporcionar a los alumnos conocimientos verdaderos o absolutos sino propiciar situaciones de aprendizaje en las que sean capaces de emitir y contrastar hipótesis de trabajo, además de promover y cambiar ciertas actitudes, los EVA facilitan la creación de ambientes enriquecidos con excelentes resultados en el desarrollo de las habilidades cognitivas.

Por tanto, la conjunción de métodos constructivistas y TIC deviene en una alternativa educativa poderosa en cuanto a la formación de profesionales competentes que tengan que desarrollar tareas cognoscitivas complejas. El uso de ambientes de simulación y EVA no se presenta como una alternativa pasajera, sino que son interpretados como instrumentos que facilitan la investigación por parte de los alumnos 
y suponen un cambio cualitativo en la forma de entender la enseñanza, ya que facilitan el aprendizaje. Los resultados obtenidos del uso de entornos de aprendizaje basados en simulaciones interactivas orientadas al tratamiento de problemas demuestran que éste planteamiento metodológico contribuye a la mejora del aprendizaje y al aumento en la confianza depositada en las respuestas (Barneto y col., 2008).

En este artículo nos centramos en el uso de EVA para la enseñanza de las ingenierías, campo en el que no se suele aprovechar la potencialidad de estos recursos, en los que el propio entorno de aprendizaje ha de proporcionar al alumno los escenarios y condiciones necesarias (normalmente simuladas) a utilizar en la confección de proyectos específicos; por ejemplo, que el propio EVA contenga el lenguaje en el que los proyectos deban ser programados, o los servicios que el alumno pueda configurar para desarrollar aplicaciones web. En particular, enfocamos el estudio hacia la utilización de estos EVA para la evaluación del desarrollo de competencias, centrando nuestra atención, en esta ocasión, en las competencias profesionales, ya que la utilización de los EVA permite la evaluación de competencias que se presentan como elementos de un proceso que conducen a un producto final, por lo que se centra la atención en una evaluación procesual más que de una evaluación final.

\section{Objetivo}

Partimos del supuesto de que el uso de EVA favorece el desarrollo de competencias profesionales y planteamos el siguiente objetivo:

- Diseñar y validar un instrumento de evaluación de competencias profesionales en el contexto universitario, aplicado mediante el uso de un EVA.

\section{Metodología}

El currículo de la carrera de Ingeniería en Informática se divide en áreas que comprenden materias afines entre sí. Existen áreas tales como Programación Básica, Programación Avanzada, Sistemas Distribuidos heterogéneos e Ingeniería de Software, entre otras. La asignatura "Taller de Desarrollo de Proyectos III", en la que se centra este trabajo, se dicta para el sexto curso de la Carrera de Ingeniería en Informática y se encuadra en el área de Ingeniería de Software.

Las competencias genéricas que se proponen para la asignatura se han elaborado teniendo en cuenta: a) las competencias en ingeniería que se recogen en los documentos del Consejo Federal de Decanos de Ingeniería (CONFEDI), en los que se definen las competencias del Ingeniero Argentino; b) la "Declaración de Valparaíso", que define las competencias del Ingeniero Iberoamericano; y c) el Proyecto Tuning para América Latina. Se procede a la selección de las competencias genéricas que tienen incidencia directa en el desarrollo de las competencias profesionales propias de la asignatura, tomando en consideración el tema central de la misma: la evolución de los diferentes tipos de arquitectura hasta el devenir de SOA (acrónimo, en inglés, de Arquitectura Orientada a Servicios).

Para evaluar el desarrollo de estas competencias genéricas nos basamos en el uso de rúbricas, instrumentos de evaluación que se definen como "descriptores cualitativos que establecen la naturaleza de un desempeño" (Simón y col., 2001). En consecuencia, son "instrumentos de medición en los cuales se establecen criterios y estándares por niveles, mediante la disposición de escalas, que permiten determinar la calidad de la ejecución de los estudiantes en tareas específicas" (Vera Vélez, 2008). 
Del proceso descrito surge la propuesta de competencias genéricas, a las que se asigna un código para referirnos a ellas en adelante (Tabla I). A partir del análisis detallado de estas competencias bajo el punto de vista de los 4 pilares del conocimiento (Delors, 1989) o tipos de saberes, se definen los elementos de la rúbrica.

Para ello, se realiza una consulta a profesores del área de Ingeniería de Software, a la que pertenece la materia Taller de Desarrollo de Proyectos III, con el propósito de establecer elementos que resulten de algún modo consensuados con otros expertos en la disciplina. La Tabla I recoge los elementos de rúbrica que finalmente se asocian a cada competencia genérica.

Tabla I. Elementos de rúbrica

\begin{tabular}{|c|c|}
\hline Competencia genérica & Elementos de rúbrica \\
\hline CT1. Arquitectura & $\begin{array}{l}\text { ER1.Caracterizar la evolución de las diferentes arquitecturas de software. } \\
\text { ER5.Controlar que ningún aspecto o decisión arquitectural dependan de un } \\
\text { determinado producto. }\end{array}$ \\
\hline CT2. Análisis & $\begin{array}{l}\text { ER18.Investigar los distintos autores y sus propuestas sobre el desarrollo de } \\
\text { proyectos. } \\
\text { ER2.Planificar el desarrollo de un proyecto }\end{array}$ \\
\hline CT3. Aplicación de conceptos & ER3.Aplicar los conceptos teóricos de sistemas en el desarrollo de proyectos. \\
\hline CT4. Calidad & ER4. Determinar la aplicación con un determinado estándar. \\
\hline CT5. Creatividad & $\begin{array}{l}\text { ER7. Reutilizar módulos. } \\
\text { ER12.Usar componentes de software implementados por terceros que utilicen } \\
\quad \text { las técnicas mencionadas. } \\
\text { ER13.Integrar las distintas componentes de un módulo. } \\
\text { ER14.Integrar módulos. } \\
\text { ER15.Caracterizar el impacto de un módulo dentro de una aplicación. }\end{array}$ \\
\hline CT6. Metodología & $\begin{array}{l}\text { ER10.Aplicar las técnicas básicas de uso más frecuente definidas como } \\
\text { buenas prácticas de programación. }\end{array}$ \\
\hline CT7. Testeo & ER20.Operar herramientas básicas de testeo de software \\
\hline CT8. Innovación & ER19.Clasificar Los distintos tipos de literatura científica. \\
\hline $\begin{array}{l}\text { CT9. Documentación - } \\
\text { Presentación }\end{array}$ & $\begin{array}{l}\text { ER16.Operar las herramientas informáticas básicas de mayor relevancia en el } \\
\text { ámbito de los procesos de Desarrollo de Proyectos de software. }\end{array}$ \\
\hline CT10. Código & ER9. Programar un módulo. \\
\hline CT11. Gestión & $\begin{array}{l}\text { ER17.Clasificar las herramientas informáticas básicas de mayor relevancia en } \\
\text { el ámbito de los procesos de Desarrollo de Proyectos. }\end{array}$ \\
\hline CT12. Despliegue & ER21.Utilizar técnicas y herramientas para el despliegue de la aplicación. \\
\hline CT13. Compromiso & ER23.Comprometerse éticamente \\
\hline $\begin{array}{l}\text { CT14. Administración de } \\
\text { proyectos }\end{array}$ & $\begin{array}{l}\text { ER22.Ejecutar e implementar de forma práctica todas las tareas que se llevan } \\
\text { a cabo en el proceso de desarrollo de software. }\end{array}$ \\
\hline CT15. Implementación & $\begin{array}{l}\text { ER11.Implementar componentes de software que utilicen las técnicas } \\
\text { mencionadas. }\end{array}$ \\
\hline CT16. Divulgación & $\begin{array}{l}\text { ER24.Desarrollar capacidades de divulgación frente a un público } \\
\text { especializado y no especializado. }\end{array}$ \\
\hline CT17. Diseño & $\begin{array}{l}\text { ER6. Determinar los requerimientos funcionales y no funcionales. } \\
\text { ER8. Diseñar módulos. }\end{array}$ \\
\hline
\end{tabular}

Definidos los elementos de rúbrica y asociados estos a cada competencia, se recurrió a un grupo de 10 expertos para su validación. Se seleccionan, atendiendo al doble criterio de poseer título de Doctor o Magister en Educación o en Enseñanza, e impartir asignaturas en el área. Se seleccionaron, pues, aquellos profesores que teniendo posgrado en enseñanza tenían dominio técnico de la disciplina, pues son profesores del Departamento de Computación de la Facultad de Ingeniería de la Universidad de Buenos Aires. En sólo dos de los casos se trató de docentes con maestría disciplinar en el campo técnico, pero en estos dos casos se seleccionaron aquellos que poseían 
cursos de especialización en docencia universitaria. Se les proporcionó un cuestionario de validación de los elementos de rúbrica (Anexo I).

El criterio para seleccionar o descartar los elementos de la rúbrica es que al menos tres cuartos de los expertos encuestados (ocho de los 10, en nuestro caso) valoraran el elemento de rúbrica como "muy adecuado" (valor 4) o "perfectamente adecuado" (valor 5). El criterio para valorar si el elemento de rúbrica era comprensible es que al menos tres cuartos de los expertos encuestados valoraran la expresión "se entiende bastante" (valor 3) o "se entiende perfectamente" (valor 4).

\section{Resultados}

Se presentan los resultados en función de los bloques del cuestionario de validación por expertos (Anexo 1):

\section{A. ¿El elemento de rúbrica es adecuado a la competencia a medir?}

La Tabla II muestra las frecuencias de respuesta de los expertos encuestados a la adecuación de los elementos de rúbrica asignados a las competencias genéricas. Con el criterio comentado, los elementos a eliminar de la propuesta inicial son ER1, ER2, ER7, ER12 y ER15.

Tabla II. Frecuencias de respuesta ante adecuación de los ER asignados a las CG

\begin{tabular}{|c|c|c|c|c|c|c|c|c|c|c|c|c|c|c|c|c|c|c|c|c|c|c|c|c|c|}
\hline \multirow{2}{*}{\multicolumn{2}{|c|}{$\begin{array}{c}\mathrm{CG} \\
\mathrm{ER}\end{array}$}} & \multicolumn{2}{|c|}{1} & \multicolumn{2}{|c|}{2} & C & 4 & \multicolumn{5}{|c|}{5} & 6 & 7 & 8 & 9 & 10 & 11 & 12 & 13 & 14 & 15 & 16 & \multicolumn{2}{|c|}{17} \\
\hline & & 1 & 5 & 18 & 2 & 3 & 4 & 7 & 12 & 13 & 14 & 15 & 10 & 20 & 19 & 16 & 9 & 17 & 21 & 23 & 22 & 11 & 24 & 6 & 8 \\
\hline \multirow{5}{*}{ 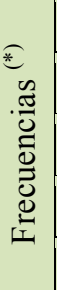 } & 1 & 0 & 0 & 0 & 0 & 0 & 0 & 0 & 0 & 0 & 0 & 0 & 0 & 1 & 0 & 0 & 0 & 0 & 0 & 0 & 0 & 0 & 0 & 0 & 0 \\
\hline & 2 & 1 & 0 & 0 & 1 & 0 & 0 & 0 & 1 & 0 & 1 & 0 & 1 & 0 & 1 & 0 & 0 & 0 & 0 & 0 & 0 & 0 & 0 & 0 & 0 \\
\hline & 3 & 2 & 0 & 2 & 4 & 0 & 0 & 4 & 5 & 0 & 0 & 3 & 0 & 0 & 0 & 1 & 0 & 0 & 0 & 0 & 0 & 0 & 1 & 0 & 0 \\
\hline & 4 & 4 & 6 & 3 & 3 & 3 & 4 & 5 & 3 & 6 & 6 & 5 & 3 & 3 & 6 & 3 & 1 & 3 & 1 & 3 & 5 & 2 & 2 & 1 & 3 \\
\hline & 5 & 3 & 4 & 5 & 2 & 7 & 6 & 1 & 1 & 4 & 3 & 2 & 6 & 6 & 3 & 6 & 9 & 7 & 9 & 7 & 5 & 8 & 7 & 9 & 7 \\
\hline
\end{tabular}

${ }^{(*)}$ 1. Poco / 2. Escasamente / 3. Bastante / 4. Muy / 5. Perfectamente

\section{B. ¿ Se entiende como está expresado el elemento de rúbrica?}

La Tabla III muestra las frecuencias de respuesta de los expertos ante la adecuación de la expresión de los elementos de rúbrica. No se estima la necesidad de modificar la expresión de ningún elemento de rúbrica.

Tabla III. Frecuencias de respuesta sobre expresión de los ER

\begin{tabular}{|c|c|c|c|c|c|c|c|c|c|c|c|c|c|c|c|c|c|c|c|c|c|c|c|c|c|}
\hline \multirow{2}{*}{\multicolumn{2}{|c|}{$\frac{\mathrm{CG}}{\mathrm{ER}}$}} & \multicolumn{2}{|c|}{1} & \multicolumn{2}{|c|}{2} & 3 & 4 & \multicolumn{5}{|c|}{5} & 6 & 7 & 8 & 9 & 10 & 11 & 12 & 13 & 14 & 15 & 16 & \multicolumn{2}{|c|}{17} \\
\hline & & 1 & 5 & 18 & 2 & 3 & 4 & 7 & 12 & 13 & 14 & 15 & 10 & 20 & 19 & 16 & 9 & 17 & 21 & 23 & 22 & 11 & 24 & 6 & 8 \\
\hline \multirow{4}{*}{ 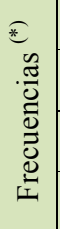 } & 1 & 0 & 0 & 0 & 0 & 0 & 0 & 0 & 0 & 0 & 0 & 0 & 0 & 0 & 0 & 0 & 0 & 0 & 0 & 0 & 0 & 0 & 0 & 0 & 1 \\
\hline & 2 & 0 & 1 & 0 & 0 & 0 & 0 & 0 & 0 & 0 & 1 & 1 & 0 & 0 & 1 & 0 & 0 & 1 & 0 & 0 & 0 & 0 & 0 & 0 & 0 \\
\hline & 3 & 4 & 6 & 3 & 3 & 2 & 4 & 2 & 4 & 2 & 2 & 5 & 1 & 1 & 0 & 6 & 2 & 6 & 0 & 1 & 5 & 3 & 2 & 1 & 0 \\
\hline & 4 & 6 & 3 & 7 & 7 & 8 & 6 & 8 & 6 & 8 & 7 & 4 & 9 & 9 & 9 & 4 & 8 & 3 & 10 & 9 & 5 & 7 & 8 & 9 & 9 \\
\hline
\end{tabular}

${ }^{(*)}$ 1. No / 2. Poco / 3. Bastante / 4. Perfectamente 


\section{Propuestas de mejora}

La Tabla IV muestra los elementos de rúbrica para los que algunos expertos realizan propuestas de mejora.

Tabla IV. Elementos de rúbrica para los que se propone mejora

\begin{tabular}{|c|c|c|c|c|c|c|c|c|c|c|c|c|c|c|c|c|c|c|c|c|c|c|c|c|c|}
\hline \multirow{2}{*}{\multicolumn{2}{|c|}{$\frac{C G}{\text { ER }}$}} & \multicolumn{2}{|c|}{1} & \multicolumn{2}{|c|}{2} & 3 & 4 & \multicolumn{5}{|c|}{5} & 6 & 7 & 8 & 9 & 10 & 11 & 12 & 13 & 14 & 15 & 16 & \multicolumn{2}{|c|}{17} \\
\hline & & 1 & 5 & 18 & 2 & 3 & 4 & 7 & 12 & 13 & 14 & 15 & 10 & 20 & 19 & 16 & 9 & 17 & 21 & 23 & 22 & 11 & 24 & 6 & 8 \\
\hline \multirow{5}{*}{ 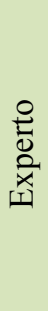 } & 1 & & & & $x$ & & & & & & & $X$ & & $x$ & & & & & & & & & & & \\
\hline & 4 & & $x$ & & & & & & & & & & & & & & & & & & & & & & $X$ \\
\hline & 7 & & & & & & & & & & & & $x$ & & $x$ & & & & & & & & & & \\
\hline & 9 & & & & & & & & $X$ & & & & & & & & & $x$ & & & & & & & \\
\hline & 10 & $X$ & & & & & & & & & $x$ & & & & & & & & & & & & & & \\
\hline
\end{tabular}

Como se puede apreciar en la tabla, ningún elemento de rúbrica recibe propuestas de mejora por parte de más de un experto. No obstante, se procede el análisis individual de las propuestas con la intención de mejorar al máximo el instrumento de evaluación.

La Tabla $V$ muestra las propuestas realizadas por los expertos y las decisiones que se adoptan al respecto, en relación con los elementos de rúbrica no eliminados.

Tabla V. Propuestas de mejora para elementos de rúbrica no eliminados

\begin{tabular}{|c|c|c|c|}
\hline Experto & $\mathbf{E R}$ & Propuesta & Decisión \\
\hline $\mathbf{1}$ & $\mathbf{2 0}$ & $\begin{array}{c}\text { Hay que aplicar una metodología de } \\
\text { testeo }\end{array}$ & $\begin{array}{c}\text { Se desestima. La idea queda incluida en el } \\
\text { enunciado original }\end{array}$ \\
\hline \multirow{2}{*}{$\mathbf{4}$} & $\mathbf{5}$ & $\begin{array}{c}\text { Identificar el problema con un tipo en } \\
\text { particular de arquitectura }\end{array}$ & $\begin{array}{c}\text { Se desestima. La propuesta no conserva el } \\
\text { sentido original del enunciado }\end{array}$ \\
\cline { 2 - 5 } & $\mathbf{8}$ & $\begin{array}{c}\text { Diseñar módulos con alta cohesión y } \\
\text { bajo acoplamiento }\end{array}$ & $\begin{array}{c}\text { Se acepta. El enunciado propuesto enuncia la } \\
\text { forma correcta del diseño de módulos. }\end{array}$ \\
\hline \multirow{2}{*}{7} & $\mathbf{1 0}$ & Usar buenas prácticas y estándares & $\begin{array}{c}\text { Se acepta. Es una forma más concreta del } \\
\text { enunciado original }\end{array}$ \\
\cline { 2 - 5 } & $\mathbf{1 9}$ & $\begin{array}{c}\text { Poner en práctica los conceptos } \\
\text { planteados en literatura científica }\end{array}$ & $\begin{array}{c}\text { Se acepta. Parece más adecuado "poner en } \\
\text { práctica" que "clasificar" }\end{array}$ \\
\hline $\mathbf{9}$ & $\mathbf{1 7}$ & $\begin{array}{c}\text { Se sugiere reemplazar "clasificar" por } \\
\text { "utilizar" }\end{array}$ & $\begin{array}{c}\text { Se acepta. Es más adecuado "utilizar" que } \\
\text { "clasificar" }\end{array}$ \\
\hline $\mathbf{1 0}$ & $\mathbf{1 4}$ & Integrar Módulos creativamente & $\begin{array}{c}\text { Se desestima. La creatividad queda definida por } \\
\text { el bloque de CT al que pertenece. }\end{array}$ \\
\hline
\end{tabular}

\section{D. ¿Considera que algún elemento de rúbrica es innecesario o sobra?}

Solo tres expertos ofrecen su opinión al respecto: el experto 1 considera que sobra ER1, el experto 4 que sobra ER10 y el experto 10 que sobra ER2.

Los elementos ER1 y ER2 ya han sido eliminados por no ser considerados "muy adecuados" o "perfectamente adecuados" "para la competencia transversal a evaluar (Tabla I). Respecto a ER10, se decide mantenerlo por ser un solo experto el que sugiere su eliminación.

\section{E. ¿Considera que falta o sería necesario agregar algún elemento de rúbrica?}


En este apartado los expertos que ofrecen opinión son los mismos que en el apartado anterior.

El experto 1 considera que a la competencia genérica CG2 habría que añadirle un elemento de rúbrica sobre "etapa de análisis", el experto 4 sugiere añadir a la competencia CG6 el elemento "aplicar la metodología más adecuada a la arquitectura" y el experto 10 considera que habría que añadir a la competencia CG1 el elemento "conoce los diferentes modelos de Ciclo de Vida".

Se decide desestimar las tres recomendaciones al considerar que están intrínsecamente contenidas en las competencias genéricas a las que referencian.

\section{Conclusiones}

La evaluación de competencias no es tarea fácil. Si partimos del hecho de que, por la propia definición de competencia, para evaluarlas deberíamos situar al alumnado en diferentes situaciones y contextos, fácilmente concluiremos que esto no es así en la mayoría de las ocasiones, en las que se trabaja en un contexto de aula. Debido a ello, a cada competencia se le suelen asignar subcompetencias o capacidades directamente evaluables, y se supone que una evaluación positiva de estas nos permite asegurar el desarrollo de la competencia en cuestión.

Por otra parte, la utilización de entornos virtuales de aprendizaje parece ser adecuada para simular contextos a los que el alumnado tendrá que enfrentarse en su futuro profesional y personal, contextos a los que, de cualquier otro modo, no sería factible acercarlos.

Es por ello por lo que decidimos la utilización de estos entornos virtuales para el diseño y testeo de un instrumento de evaluación de competencias. Tras el diseño inicial de una rúbrica de evaluación en la que a cada una de las competencias se le asignan elementos de rúbrica particulares, se procede a su validación por 10 expertos y se obtiene como resultado la rúbrica cuyos elementos se presentan en la Tabla VI.

\begin{tabular}{|c|c|}
\hline \multicolumn{2}{|c|}{ Tabla VI. Elementos de rúbrica de evaluación de Competencias Genéricas } \\
\hline Competencia Genérica & Elementos de rúbrica \\
\hline CG1 Arquitectura & ER5 \\
\hline CG2 Análisis & ER18 \\
\hline CG3 Aplicación de conceptos & ER3 \\
\hline CG4 Calidad & ER4 \\
\hline CG5 Creatividad & ER13-ER14 \\
\hline CG6 Metodología & ER10 \\
\hline CG7 Testeo & ER20 \\
\hline CG8 Innovación & ER19 \\
\hline CG9 Documentación-Presentación & ER16 \\
\hline CG10 Código & ER9 \\
\hline CG11 Gestión & ER17 \\
\hline CG12 Despliegue & ER21 \\
\hline CG13 Compromiso & ER23 \\
\hline CG14 Administración de proyectos & ER22 \\
\hline CG15 Implementación & ER11 \\
\hline CG16 Divulgación & ER24 \\
\hline CG17 Diseño & ER6-ER8 \\
\hline
\end{tabular}


En el Anexo II se muestra la rúbrica completa con indicadores de logro para cada uno de sus elementos. Ahora queda probar su eficacia para la evaluación de competencias profesionales, lo que se expondrá en trabajos futuros. Al tratarse de competencias genéricas, muchos de los elementos de rúbrica que se presentan en este artículo pueden ser útiles para otras asignaturas. Incluso el proceso de elaboración de la rúbrica, esperamos, puede ser reproducido para la elaboración de otras rúbricas más específicas.

\section{Referencias}

Barneto, A.G. y Bolívar Raya, J.P. (2008). Efecto de las simulaciones interactivas sobre las concepciones de los alumnos en relación con el movimiento armónico simple. Revista Electrónica de Enseñanza de las Ciencias. 7(3).

Beneitone, P. Esaquetini, C. González J. Marty, M. Siufi, G. y Wagenaar, R. (2007). Reflexiones y perspectivas de la educación superior en América Latina. Informe final proyecto Tuning Amércia Latina 2004-2007. Bilbao, Publicaciones de la Universidad de Deusto.

Berners-Lee T. (2002). WWW: Past, present, and future. Computer, 29, 69-77.

Delors, J. (1989). La educación encierra un tesoro. Madrid: UNESCO.

Hung, D. (2002). Situated cognition and problem-based learnig:implications for learning and instructions with Tecnology, Journal of interactive Learnig research.

Medina Rivilla, A., Domínguez Garrido, MC., Sánchez Romero, C. (2013). Evaluación de las competencias de los estudiantes: modelos y técnicas para la valoración. Revista de Investigación Educativa, 31 (1), $239-255$.

Neira, K. y Bustos, H. (2014). Ambientes de aprendizaje en el modelo basado en competencias Duoc UC: Simulación clínica como Tecnología educativa. Comunicación presentada en el XIV Congreso Internacional EDUTEC 2014, Noviembre. Córdoba.

Pontes Pedrajas, A. (2005). Aplicaciones de las tecnologías de la información y de la comunicación en la educación científica. Primera parte: funciones y recursos. Revista Eureka sobre Enseñanza y Divulgación de las Ciencias. 2(1).

Raviolo, A. (2010). Simulaciones en la enseñanza de la química. VI Jornadas Internacionales y IX Jornadas Nacionales de Enseñanza Universitaria de la Química. Santa Fe.

Rodriguez, R. (2008). Un modelo de formación basado en las competencias: Hacia un nuevo paradigma en la enseñanza universitaria. Contextos educativos, 11, 131-147

Salinas, Jesús (2004). "Innovación docente y uso de las TIC en la enseñanza universitaria". Revista de Universidad y Sociedad del Conocimiento (RUSC). [artículo en línea]. UOC. Vol. 1, nº 1.

Sánchez, DI. (2007). Diseño y desarrollo de un software, para el aprendizaje de ssh (secure shell), mediante un Entorno Virtual. Bogota.

Simón, M., \& Forgette-Giroux, R. (2001). A rubric for scoring postsecondary academic skills. Practical Assessment, Research \& Evaluation, volúmen 7 , número18.

Vera Vélez, L. (2008). La rúbrica y la lista de cotejo. http://www.tecnoedu.net/lecturas/materiales/lectura10.pdf 


\section{ANEXO 1}

COMPETENCIAS GENERICAS A SER EVALUADAS EN LA ASIGNATURA

75.55 TALLER DE DESARROLLO DE PROYECTOS III CUESTIONARIO A EXPERTOS (Título de Doctor o Magister)

\begin{tabular}{|c|c|c|c|c|c|}
\hline ID. & Competencia & Elemento de Rubrica & $\begin{array}{l}\text { El Elemento de Rubrica es } \\
\text { adecuado a la competencia a } \\
\text { medir? } \\
\text { 1. Poco adecuado } \\
\text { 2. Escasamente adecuado } \\
\text { 3. Bastante adecuado } \\
\text { 4. Muy adecuado } \\
\text { 5. Perfectamente } \\
\text { adecuado }\end{array}$ & $\begin{array}{l}\text { Se entiende cómo está } \\
\text { expresado el Elemento } \\
\text { de Rubrica? } \\
\text { 1. No se entiende } \\
\text { 2. Se entiende poco } \\
\text { 3. Se entiende } \\
\text { bastante } \\
\text { 4. Se entiende } \\
\text { perfectamente }\end{array}$ & $\begin{array}{r}\text { Propuestas } \\
\text { de Mejora }\end{array}$ \\
\hline \multirow[t]{2}{*}{ CG1 } & \multirow[t]{2}{*}{ Arquitectura } & $\begin{array}{l}\text { Caracterizar la evolución } \\
\text { de las diferentes } \\
\text { arquitecturas de software. }\end{array}$ & & & \\
\hline & & $\begin{array}{l}\text { Controlar que ningún } \\
\text { aspecto o decisión } \\
\text { arquitectural dependan de } \\
\text { un determinado producto. }\end{array}$ & & & \\
\hline \multirow[t]{2}{*}{ CG2 } & \multirow[t]{2}{*}{ Análisis } & $\begin{array}{l}\text { Investigar los distintos } \\
\text { autores y sus propuestas } \\
\text { sobre el desarrollo de } \\
\text { proyectos. }\end{array}$ & & & \\
\hline & & $\begin{array}{l}\text { Planificar el desarrollo de } \\
\text { un proyecto }\end{array}$ & & & \\
\hline CG3 & $\begin{array}{l}\text { Aplicación de } \\
\text { conceptos }\end{array}$ & $\begin{array}{l}\text { Aplicar los conceptos } \\
\text { teóricos de sistemas en el } \\
\text { desarrollo de proyectos. }\end{array}$ & & & \\
\hline CG4 & Calidad & $\begin{array}{l}\text { Determinar la aplicación } \\
\text { con un determinado } \\
\text { estándar. }\end{array}$ & & & \\
\hline \multirow[t]{5}{*}{ CG5 } & \multirow[t]{5}{*}{ Creatividad } & Reutilizar módulos & & & \\
\hline & & $\begin{array}{l}\text { Usar componentes de } \\
\text { software implementados } \\
\text { por terceros que utilicen } \\
\text { las técnicas mencionadas. }\end{array}$ & & & \\
\hline & & $\begin{array}{l}\text { Integrar las distintas } \\
\text { componentes de un } \\
\text { módulo. }\end{array}$ & & & \\
\hline & & Integrar módulos. & & & \\
\hline & & $\begin{array}{l}\text { Caracterizar el impacto de } \\
\text { un módulo dentro de una } \\
\text { aplicación. }\end{array}$ & & & \\
\hline CG6 & Metodología & $\begin{array}{l}\text { Aplicar las técnicas } \\
\text { básicas de uso más } \\
\text { frecuente definidas como } \\
\text { buenas prácticas de } \\
\text { programación. }\end{array}$ & & & \\
\hline CG7 & Testing & $\begin{array}{l}\text { Operar herramientas } \\
\text { básicas de testeo de } \\
\text { software }\end{array}$ & & & \\
\hline CG8 & Innovación & $\begin{array}{l}\text { Clasificar los distintos } \\
\text { tipos de literatura } \\
\text { científica. }\end{array}$ & & & \\
\hline
\end{tabular}




\begin{tabular}{|c|c|c|c|c|}
\hline CG9 & $\begin{array}{l}\text { Documenta } \\
\text { ción - Presentación }\end{array}$ & \begin{tabular}{|l|} 
Operar las herramientas \\
informáticas básicas de \\
mayor relevancia en el \\
ámbito de los procesos de \\
Desarrollo de Proyectos \\
de software. \\
\end{tabular} & & \\
\hline $\begin{array}{l}\mathrm{CG} \\
10\end{array}$ & Código & Programar un módulo. & & \\
\hline $\begin{array}{l}\mathrm{CG} \\
11\end{array}$ & Gestión & $\begin{array}{l}\text { Clasificar las } \\
\text { herramientas informáticas } \\
\text { básicas de mayor } \\
\text { relevancia en el ámbito de } \\
\text { los procesos de } \\
\text { Desarrollo de Proyectos. }\end{array}$ & & \\
\hline $\begin{array}{l}\mathrm{CG} \\
12\end{array}$ & Despliegue & $\begin{array}{l}\text { Utilizar técnicas y } \\
\text { herramientas para el } \\
\text { despliegue de la } \\
\text { aplicación. }\end{array}$ & & \\
\hline $\begin{array}{l}\text { CG } \\
13 \\
\end{array}$ & Compromiso & $\begin{array}{l}\text { Comprometerse } \\
\text { éticamente }\end{array}$ & & \\
\hline $\begin{array}{l}\text { CG } \\
14\end{array}$ & $\begin{array}{l}\text { Administración de } \\
\text { proyectos }\end{array}$ & $\begin{array}{l}\text { Ejecutar e implementar de } \\
\text { forma práctica todas las } \\
\text { tareas que se llevan a } \\
\text { cabo en el proceso de } \\
\text { desarrollo de software. } \\
\end{array}$ & & \\
\hline $\begin{array}{l}\text { CG } \\
15\end{array}$ & $\begin{array}{l}\text { Implementa } \\
\text { ción }\end{array}$ & $\begin{array}{l}\text { Implementar } \\
\text { componentes de software } \\
\text { que utilicen las técnicas } \\
\text { mencionadas. }\end{array}$ & & \\
\hline $\begin{array}{l}\text { CG } \\
16\end{array}$ & $\begin{array}{l}\text { Comunica } \\
\text { ción }\end{array}$ & $\begin{array}{l}\text { Desarrollar capacidades } \\
\text { de divulgación frente a un } \\
\text { público especializado } \\
\text { y no especializado. } \\
\end{array}$ & & \\
\hline \multirow[t]{2}{*}{$\begin{array}{l}\text { CG } \\
17\end{array}$} & \multirow[t]{2}{*}{ Diseño } & $\begin{array}{l}\text { Determinar los } \\
\text { requerimientos } \\
\text { funcionales y no } \\
\text { funcionales. } \\
\end{array}$ & & \\
\hline & & Diseñar módulos. & & \\
\hline
\end{tabular}

\section{CONSIDERA QUE ALGÚN ELEMENTO DE RUBRICA ES INNECESARIO O SOBRA?}

CONSIDERA QUE FALTA O SERÍA NECESARIO AGREGAR ALGÚN ELEMENTO DE RUBRICA?

QUITARÍA / AGREGARÍA ALGUNA COMPETENCIA GENÉRICA EN RELACIÓN A LA ASIGNATURA 75.55 TALLER DE DESARROLLO DE PROYECTOS III? 
ANEXO 2.

\section{RÚBRICA DE EVALUACIÓN DEL DESARROLLO DE COMPETENCIAS} GENÉRICAS

\begin{tabular}{|c|c|c|c|}
\hline Excelente & Correcto & Insuficiente & Muy Pobre \\
\hline $\begin{array}{l}\text { Nivel excepcional de } \\
\text { desempeño, excediendo } \\
\text { todo lo esperado. } \\
\text { Propone o desarrolla nuevas acciones. } \\
\text { Respuesta completa. } \\
\text { Explicaciones claras del concepto. } \\
\text { Identifica todos los } \\
\text { elementos importantes. } \\
\text { Provee buenos ejemplos. } \\
\text { Ofrece información que va más allá de } \\
\text { lo enseñado en clase. }\end{array}$ & $\begin{array}{l}\text { Nivel de desempeño cercano de lo } \\
\text { esperado } \\
\text { Presenta frecuencia baja de errores. } \\
\text { Comprensión del problema. } \\
\text { Algunos de los requerimientos de la tarea } \\
\text { faltan en la respuesta. } \\
\text { No logra demostrar que aplica el } \\
\text { concepto. } \\
\text { Omite algunos elementos, si bien } \\
\text { identifica algunos de ellos. } \\
\text { Ofrece información relacionada a lo } \\
\text { enseñado en clase. }\end{array}$ & $\begin{array}{l}\text { No satisface prácticamente } \\
\text { nada de los requerimientos de } \\
\text { desempeño. } \\
\text { No comprende el problema. } \\
\text { No aplica los requerimientos } \\
\text { para la tarea. } \\
\text { Omite las partes fundamentales } \\
\text { del concepto. }\end{array}$ & $\begin{array}{l}\text { No realiza la } \\
\text { actividad. } \\
\text { No intenta } \\
\text { elaborarla }\end{array}$ \\
\hline
\end{tabular}

\begin{tabular}{|c|c|c|c|c|}
\hline ITEMS & EXCELENTE & CORRECTO & INSUFICIENTE & MUY POBRE \\
\hline \multicolumn{5}{|l|}{ CG1Arquitectura } \\
\hline $\begin{array}{l}\text { ER1.-Caracterizar la } \\
\text { evolución de las } \\
\text { diferentes } \\
\text { arquitecturas de } \\
\text { software. }\end{array}$ & $\begin{array}{l}\text { Las decisiones sobre la } \\
\text { aplicabilidad de un } \\
\text { determinado proceso de } \\
\text { desarrollo de software son } \\
\text { acertadas y correctas en } \\
\text { todos los casos. } \\
\text { Demuestra conocimiento } \\
\text { del por qué de dichas } \\
\text { decisiones. }\end{array}$ & $\begin{array}{l}\text { Las decisiones sobre la } \\
\text { aplicabilidad de un } \\
\text { determinado proceso de } \\
\text { desarrollo de software son } \\
\text { en la mayoría de los casos } \\
\text { acertadas. }\end{array}$ & $\begin{array}{l}\text { Las decisiones sobre la } \\
\text { aplicabilidad de un } \\
\text { determinado proceso de } \\
\text { desarrollo de software son } \\
\text { en algunos de los casos } \\
\text { acertadas. Omite las partes } \\
\text { fundamentales del } \\
\text { concepto. }\end{array}$ & $\begin{array}{l}\text { No son acertadas la } \\
\text { decisiones sobre la } \\
\text { aplicabilidad de un } \\
\text { determinado proceso } \\
\text { de desarrollo de } \\
\text { software. No } \\
\text { demuestra } \\
\text { conocimiento del por } \\
\text { qué son aplicadas } \\
\text { dichas decisiones. } \\
\text { No realiza la actividad. }\end{array}$ \\
\hline $\begin{array}{l}\text { ER5.-Controlar que } \\
\text { ningún aspecto o } \\
\text { decisión arquitectural } \\
\text { dependan de un } \\
\text { determinado } \\
\text { producto. }\end{array}$ & $\begin{array}{l}\text { Capaz de controlar en } \\
\text { todas las situaciones } \\
\text { propuestas que ningún } \\
\text { aspecto o decisión } \\
\text { arquitectural dependan de } \\
\text { un determinado producto. }\end{array}$ & $\begin{array}{l}\text { Capaz de controlar en la } \\
\text { mayoría de las situaciones } \\
\text { propuestas que ningún } \\
\text { aspecto o decisión } \\
\text { arquitectural dependan de } \\
\text { un determinado producto. }\end{array}$ & $\begin{array}{l}\text { No comprende el Problema. } \\
\text { Controla en algunas de las } \\
\text { situaciones propuestas que } \\
\text { ningún aspecto o decisión } \\
\text { arquitectural dependan de } \\
\text { un determinado producto. }\end{array}$ & $\begin{array}{l}\text { No intenta siquiera una } \\
\text { elaboración del } \\
\text { problema. }\end{array}$ \\
\hline \multicolumn{5}{|l|}{ CG2Análisis } \\
\hline $\begin{array}{l}\text { ER18.-Investigar los } \\
\text { distintos autores y sus } \\
\text { propuestas sobre el } \\
\text { desarrollo de } \\
\text { proyectos. }\end{array}$ & $\begin{array}{l}\text { Demuestra que su } \\
\text { investigación, los distintos } \\
\text { autores y sus propuestas } \\
\text { sobre el desarrollo de } \\
\text { proyectos, es muy } \\
\text { completa y abarcativa. }\end{array}$ & $\begin{array}{l}\text { Demuestra que su } \\
\text { investigación, los distintos } \\
\text { autores y sus propuestas } \\
\text { sobre el desarrollo de } \\
\text { proyectos, es buena. }\end{array}$ & $\begin{array}{l}\text { Su investigación, los } \\
\text { distintos autores y sus } \\
\text { propuestas sobre el } \\
\text { desarrollo de proyectos, es } \\
\text { deficiente. }\end{array}$ & $\begin{array}{l}\text { No realiza la actividad. } \\
\text { No intenta la } \\
\text { elaboración del } \\
\text { problema. }\end{array}$ \\
\hline $\begin{array}{l}\text { ER2.-Planificar el } \\
\text { desarrollo de un }\end{array}$ & $\begin{array}{l}\text { La aplicación de un ciclo } \\
\text { de vida de un determinado }\end{array}$ & $\begin{array}{l}\text { La aplicación de un ciclo } \\
\text { de vida de un determinado }\end{array}$ & $\begin{array}{l}\text { La aplicación de un ciclo de } \\
\text { vida de un determinado }\end{array}$ & $\begin{array}{l}\text { No demuestra } \\
\text { conocimientos sobre la }\end{array}$ \\
\hline
\end{tabular}




\begin{tabular}{|c|c|c|c|c|}
\hline proyecto & $\begin{array}{l}\text { proyecto de desarrollo de } \\
\text { software, es acertado en } \\
\text { la totalidad de los casos a } \\
\text { los cuales el alumno es } \\
\text { expuesto. Se demuestran } \\
\text { conocimientos que } \\
\text { exceden la currícula de la } \\
\text { materia. }\end{array}$ & $\begin{array}{l}\text { proyecto de desarrollo de } \\
\text { software, es acertado en la } \\
\text { mayoría de los casos a los } \\
\text { cuales el alumno es } \\
\text { expuesto. Se demuestran } \\
\text { conocimientos necesarios } \\
\text { para la currícula de la } \\
\text { materia. }\end{array}$ & $\begin{array}{l}\text { proyecto de desarrollo de } \\
\text { software, es acertado sólo } \\
\text { en algunos de los casos. Las } \\
\text { decisiones de aplicabilidad } \\
\text { no son suficientes. }\end{array}$ & $\begin{array}{l}\text { aplicación de un ciclo } \\
\text { de vida de un } \\
\text { determinado proyecto } \\
\text { de software. } \\
\text { No realiza la actividad. }\end{array}$ \\
\hline \multicolumn{5}{|c|}{ CG3Aplicación de Conceptos } \\
\hline $\begin{array}{l}\text { ER3.Aplicar los } \\
\text { conceptos teóricos de } \\
\text { sistemas en el } \\
\text { desarrollo de } \\
\text { proyectos. }\end{array}$ & \begin{tabular}{|l|} 
El control realizado sobre \\
la aplicabilidad de los \\
conceptos teóricos es \\
completo en el total de los \\
casos y excede el \\
conocimiento requerido \\
para la materia.
\end{tabular} & $\begin{array}{l}\text { El control realizado sobre } \\
\text { la aplicabilidad de los } \\
\text { conceptos teóricos es } \\
\text { completo en la mayoría de } \\
\text { los casos, comprende el } \\
\text { problema omitiendo } \\
\text { algunos elementos e } \\
\text { identificando algunos } \\
\text { otros. }\end{array}$ & $\begin{array}{l}\text { No comprende el problema. } \\
\text { El control sobre la correcta } \\
\text { aplicación de los conceptos } \\
\text { teóricos no satisface los } \\
\text { requerimientos mínimos de } \\
\text { desempeño. }\end{array}$ & $\begin{array}{l}\text { No controla la } \\
\text { aplicabilidad de los } \\
\text { conceptos teóricos. No } \\
\text { intenta siquiera una } \\
\text { elaboración del } \\
\text { problema. }\end{array}$ \\
\hline \multicolumn{5}{|l|}{ CG4 Calidad } \\
\hline $\begin{array}{l}\text { ER4.- Determinar la } \\
\text { aplicación con un } \\
\text { determinado } \\
\text { estándar. }\end{array}$ & $\begin{array}{l}\text { De la totalidad de } \\
\text { estándares a aplicar } \\
\text { selecciona el correcto en } \\
\text { todos los casos } \\
\text { planteados. Demuestra } \\
\text { conocimientos para la } \\
\text { determinación de cual } \\
\text { estándar aplicar en todos } \\
\text { los casos. }\end{array}$ & $\begin{array}{l}\text { De la totalidad de } \\
\text { estándares a aplicar } \\
\text { selecciona el correcto en la } \\
\text { mayoría de los casos } \\
\text { planteados. Demuestra } \\
\text { conocimientos para la } \\
\text { determinación de cual } \\
\text { estándar aplicar en cada } \\
\text { caso. }\end{array}$ & $\begin{array}{l}\text { De la totalidad de } \\
\text { estándares a aplicar } \\
\text { selecciona el correcto en } \\
\text { algunos de los casos } \\
\text { planteados. No demuestra } \\
\text { un acabado conocimiento } \\
\text { para la determinación de } \\
\text { cual estándar aplicar en } \\
\text { cada caso. }\end{array}$ & $\begin{array}{l}\text { No determina la } \\
\text { aplicación de ninguno } \\
\text { de los estándares } \\
\text { propuestos. No intenta } \\
\text { siquiera una } \\
\text { elaboración del } \\
\text { problema. No intenta } \\
\text { siquiera una } \\
\text { elaboración del } \\
\text { problema. }\end{array}$ \\
\hline \multicolumn{5}{|l|}{ CG5 Creatividad } \\
\hline $\begin{array}{l}\text { ER7.- Reutilizar } \\
\text { módulos. }\end{array}$ & $\begin{array}{l}\text { Demuestra gran habilidad } \\
\text { en la reutilización de } \\
\text { módulos en la totalidad de } \\
\text { los ejercicios propuestos. }\end{array}$ & $\begin{array}{l}\text { Reutiliza módulos en la } \\
\text { mayoría de las situaciones } \\
\text { problemáticas planteadas. }\end{array}$ & $\begin{array}{l}\text { Reutiliza módulos } \\
\text { solamente en algunas de las } \\
\text { situaciones problemáticas } \\
\text { planteadas. } \\
\text { No comprende el problema } \\
\text { omitiendo partes } \\
\text { fundamentales de los } \\
\text { conceptos. }\end{array}$ & $\begin{array}{l}\text { No realiza la actividad. } \\
\text { No intenta la } \\
\text { elaboración del } \\
\text { problema. }\end{array}$ \\
\hline $\begin{array}{l}\text { ER12.-Usar } \\
\text { componentes de } \\
\text { software } \\
\text { implementados por } \\
\text { terceros que utilicen } \\
\text { las técnicas } \\
\text { mencionadas. }\end{array}$ & $\begin{array}{l}\text { En todas las situaciones } \\
\text { problemáticas planteadas } \\
\text { se utilizan componentes } \\
\text { de software } \\
\text { implementados por } \\
\text { terceros que utilicen las } \\
\text { técnicas mencionadas en } \\
\text { clase, demostrando un } \\
\text { excelente conocimiento de } \\
\text { los mismos. }\end{array}$ & $\begin{array}{l}\text { En la mayoría las } \\
\text { situaciones problemáticas } \\
\text { planteadas se utilizan } \\
\text { componentes de software } \\
\text { implementados por } \\
\text { terceros que utilicen las } \\
\text { técnicas mencionadas en } \\
\text { clase, demostrando un } \\
\text { buen conocimiento de los } \\
\text { mismos. }\end{array}$ & $\begin{array}{l}\text { En las situaciones } \\
\text { problemáticas planteadas } \\
\text { no se utilizan componentes } \\
\text { de software implementados } \\
\text { por terceros que utilicen las } \\
\text { técnicas mencionadas en } \\
\text { clase, demostrando } \\
\text { desconocer el problema. }\end{array}$ & $\begin{array}{l}\text { No realiza la actividad. } \\
\text { No intenta la } \\
\text { elaboración del } \\
\text { problema. }\end{array}$ \\
\hline $\begin{array}{l}\text { ER13.-Integrar las } \\
\text { distintas } \\
\text { componentes de un } \\
\text { módulo. }\end{array}$ & $\begin{array}{l}\text { La integración de las } \\
\text { distintas componentes se } \\
\text { realiza de manera } \\
\text { completa, acorde con las } \\
\text { especificaciones en todos } \\
\text { los ejercicios planteados. }\end{array}$ & $\begin{array}{l}\text { La integración de las } \\
\text { distintas componentes se } \\
\text { realiza de manera } \\
\text { completa, acorde con las } \\
\text { especificaciones en la } \\
\text { mayoría de los ejercicios } \\
\text { planteados. }\end{array}$ & $\begin{array}{l}\text { La integración de las } \\
\text { distintas componentes se } \\
\text { realiza de manera completa, } \\
\text { acorde con las } \\
\text { especificaciones solo en } \\
\text { algunos de los ejercicios } \\
\text { planteados. } \\
\text { No demuestra } \\
\text { conocimientos sobre el } \\
\text { tema. }\end{array}$ & $\begin{array}{l}\text { No realiza la actividad. } \\
\text { No intenta la } \\
\text { elaboración del } \\
\text { problema. }\end{array}$ \\
\hline $\begin{array}{l}\text { ER14.-Integrar } \\
\text { módulos. }\end{array}$ & $\begin{array}{l}\text { La integración de los } \\
\text { módulos es completa, } \\
\text { respeta los estándares en } \\
\text { todas las situaciones } \\
\text { propuestas, superando las }\end{array}$ & $\begin{array}{l}\text { La integración de los } \\
\text { módulos es completa, } \\
\text { respeta los estándares en } \\
\text { la mayoría las situaciones } \\
\text { propuestas, cubriendo las }\end{array}$ & $\begin{array}{l}\text { La integración de los } \\
\text { módulos es incompleta, no } \\
\text { respeta los estándares en la } \\
\text { mayoría las situaciones } \\
\text { propuestas, demuestra no }\end{array}$ & $\begin{array}{l}\text { No realiza la actividad. } \\
\text { No intenta la } \\
\text { elaboración del } \\
\text { problema. }\end{array}$ \\
\hline
\end{tabular}




\begin{tabular}{|c|c|c|c|c|}
\hline & expectativas. & expectativas. & entender el problema. & \\
\hline $\begin{array}{l}\text { ER15.-Caracterizar el } \\
\text { impacto de un } \\
\text { módulo dentro de una } \\
\text { aplicación. }\end{array}$ & $\begin{array}{l}\text { La caracterización del } \\
\text { impacto de un módulo } \\
\text { dentro de una aplicación } \\
\text { que utiliza el estándar } \\
\text { SOA, es muy completa en } \\
\text { todos los problemas } \\
\text { planteados. }\end{array}$ & $\begin{array}{l}\text { La caracterización del } \\
\text { impacto de un módulo } \\
\text { dentro de una aplicación } \\
\text { que utiliza el estándar } \\
\text { SOA, es correcta en la } \\
\text { mayoría de los problemas } \\
\text { planteados. }\end{array}$ & $\begin{array}{l}\text { La caracterización del } \\
\text { impacto de un módulo } \\
\text { dentro de una aplicación } \\
\text { que utiliza el estándar SOA, } \\
\text { es completa solo algunos de } \\
\text { los problemas planteados. }\end{array}$ & $\begin{array}{l}\text { No realiza la actividad. } \\
\text { No intenta la } \\
\text { elaboración del } \\
\text { problema. }\end{array}$ \\
\hline \multicolumn{5}{|l|}{ CG6 Metodología } \\
\hline $\begin{array}{l}\text { ER10.- Usar buenas } \\
\text { prácticas y estándares }\end{array}$ & \begin{tabular}{|l|} 
Las técnicas básicas de uso \\
más frecuente definidas \\
como buenas prácticas de \\
programación son \\
aplicadas en todas las \\
situaciones planteadas.
\end{tabular} & $\begin{array}{l}\text { Las técnicas básicas de uso } \\
\text { más frecuente definidas } \\
\text { como buenas prácticas de } \\
\text { programación son } \\
\text { aplicadas en la mayoría de } \\
\text { las situaciones planteadas. }\end{array}$ & $\begin{array}{l}\text { Las técnicas básicas de uso } \\
\text { más frecuente definidas } \\
\text { como buenas prácticas de } \\
\text { programación no son } \\
\text { aplicadas en las situaciones } \\
\text { planteadas. }\end{array}$ & $\begin{array}{l}\text { No realiza la actividad. } \\
\text { No intenta la } \\
\text { elaboración del } \\
\text { problema. }\end{array}$ \\
\hline \multicolumn{5}{|l|}{ CG7 Testeo } \\
\hline $\begin{array}{l}\text { ER20.-Operar } \\
\text { herramientas básicas } \\
\text { de testeo de software }\end{array}$ & $\begin{array}{l}\text { Los pasos de un } \\
\text { determinado proceso de } \\
\text { Evaluación son aplicados } \\
\text { en su totalidad. }\end{array}$ & $\begin{array}{l}\text { La mayoría de los pasos de } \\
\text { un determinado proceso } \\
\text { de Evaluación no son } \\
\text { aplicados en su totalidad. } \\
\text { Sólo alguno de los } \\
\text { procesos no son aplicados }\end{array}$ & $\begin{array}{l}\text { Algunos de los pasos de un } \\
\text { determinado proceso de } \\
\text { Evaluación son aplicados. } \\
\text { Muchos de los procesos no } \\
\text { son aplicados. Omite las } \\
\text { partes fundamentales del } \\
\text { concepto. }\end{array}$ & $\begin{array}{l}\text { No se aplican procesos } \\
\text { de Evaluación. } \\
\text { No realiza la actividad. }\end{array}$ \\
\hline \multicolumn{5}{|l|}{ CG8 Innovación } \\
\hline $\begin{array}{l}\text { ER19.- Poner en } \\
\text { práctica los conceptos } \\
\text { planteados en } \\
\text { literatura científica }\end{array}$ & \begin{tabular}{|l|} 
La clasificación de los \\
distintos tipos de \\
literatura científica es muy \\
completa y excede las \\
consignas propuestas.
\end{tabular} & $\begin{array}{l}\text { La clasificación de los } \\
\text { distintos tipos de literatura } \\
\text { científica es buena y } \\
\text { cumple con las consignas } \\
\text { propuestas. }\end{array}$ & $\begin{array}{l}\text { La clasificación de los } \\
\text { distintos tipos de literatura } \\
\text { científica no es muy } \\
\text { completa y no cumple con } \\
\text { las consignas propuestas. }\end{array}$ & $\begin{array}{l}\text { No realiza la actividad. } \\
\text { No intenta la } \\
\text { elaboración del } \\
\text { problema. }\end{array}$ \\
\hline \multicolumn{5}{|c|}{ CG9 Documentación-Presentación } \\
\hline $\begin{array}{l}\text { ER16.-Operar las } \\
\text { herramientas } \\
\text { informáticas básicas } \\
\text { de mayor relevancia } \\
\text { en el ámbito de los } \\
\text { procesos de } \\
\text { Desarrollo de } \\
\text { Proyectos de } \\
\text { software. }\end{array}$ & $\begin{array}{l}\text { El alumno opera todas las } \\
\text { herramientas informáticas } \\
\text { básicas de mayor } \\
\text { relevancia en el ámbito de } \\
\text { los procesos de Desarrollo } \\
\text { de Proyectos de software, } \\
\text { demostrando un excelente } \\
\text { dominio de las mismas. }\end{array}$ & $\begin{array}{l}\text { El alumno opera la } \\
\text { mayoría de las } \\
\text { herramientas informáticas } \\
\text { básicas de mayor } \\
\text { relevancia en el ámbito de } \\
\text { los procesos de Desarrollo } \\
\text { de Proyectos de software, } \\
\text { demostrando un buen } \\
\text { dominio de las mismas. }\end{array}$ & $\begin{array}{l}\text { El alumno opera una } \\
\text { reducida cantidad de las } \\
\text { herramientas informáticas } \\
\text { básicas de mayor relevancia } \\
\text { en el ámbito de los procesos } \\
\text { de Desarrollo de Proyectos } \\
\text { de software, demostrando } \\
\text { poco dominio de las mismas. }\end{array}$ & $\begin{array}{l}\text { No realiza la actividad. } \\
\text { No intenta la } \\
\text { elaboración del } \\
\text { problema. }\end{array}$ \\
\hline \multicolumn{5}{|l|}{ CG10 Código } \\
\hline $\begin{array}{l}\text { ER9.- Programar un } \\
\text { módulo. }\end{array}$ & $\begin{array}{l}\text { La programación de un } \\
\text { módulo cumple con todos } \\
\text { los requerimientos } \\
\text { necesarios, y en algunos } \\
\text { casos excede los mismos. } \\
\text { Demuestra gran } \\
\text { conocimiento sobre el } \\
\text { tema }\end{array}$ & $\begin{array}{l}\text { La programación de un } \\
\text { módulo cumple con todos } \\
\text { los requerimientos } \\
\text { necesarios en la mayoría } \\
\text { de los casos. Demuestra } \\
\text { conocimiento sobre el } \\
\text { tema }\end{array}$ & $\begin{array}{l}\text { La programación de un } \\
\text { módulo no cumple con } \\
\text { todos los requerimientos } \\
\text { necesarios, y en algunos } \\
\text { casos demuestra no } \\
\text { entender el problema. } \\
\text { Demuestra escaso } \\
\text { conocimiento sobre el tema }\end{array}$ & $\begin{array}{l}\text { No realiza la actividad. } \\
\text { No intenta la } \\
\text { elaboración del } \\
\text { problema. }\end{array}$ \\
\hline
\end{tabular}




\begin{tabular}{|c|c|c|c|c|}
\hline \multicolumn{5}{|l|}{ CG11 Gestión } \\
\hline $\begin{array}{l}\text { ER17.- Utilizar las } \\
\text { herramientas } \\
\text { informáticas básicas } \\
\text { de mayor relevancia } \\
\text { en el ámbito de los } \\
\text { procesos de } \\
\text { Desarrollo de } \\
\text { Proyectos. }\end{array}$ & $\begin{array}{l}\text { El alumno puede clasificar } \\
\text { las herramientas } \\
\text { informáticas básicas de } \\
\text { mayor relevancia en el } \\
\text { ámbito de los procesos de } \\
\text { Desarrollo de Proyectos, } \\
\text { en todos los casos } \\
\text { plateados. }\end{array}$ & $\begin{array}{l}\text { El alumno puede clasificar } \\
\text { las herramientas } \\
\text { informáticas básicas de } \\
\text { mayor relevancia en el } \\
\text { ámbito de los procesos de } \\
\text { Desarrollo de Proyectos, } \\
\text { en la mayoría de los casos } \\
\text { plateados. }\end{array}$ & $\begin{array}{l}\text { El alumno puede clasificar } \\
\text { las herramientas } \\
\text { informáticas básicas de } \\
\text { mayor relevancia en el } \\
\text { ámbito de los procesos de } \\
\text { Desarrollo de Proyectos, } \\
\text { solo en alguno de los casos } \\
\text { plateados, demostrando } \\
\text { poco dominio sobre el tema. }\end{array}$ & $\begin{array}{l}\text { No realiza la actividad. } \\
\text { No intenta la } \\
\text { elaboración del } \\
\text { problema. }\end{array}$ \\
\hline \multicolumn{5}{|l|}{ CG12 Despliegue } \\
\hline $\begin{array}{l}\text { ER21.-Utilizar técnicas } \\
\text { y herramientas para el } \\
\text { despliegue de la } \\
\text { aplicación. }\end{array}$ & $\begin{array}{l}\text { En la totalidad de los casos } \\
\text { planteados es capaz de } \\
\text { realizar el despliegue de la } \\
\text { aplicación. }\end{array}$ & $\begin{array}{l}\text { En la mayoría de los casos } \\
\text { planteados es capaz de } \\
\text { realizar el despliegue de la } \\
\text { aplicación. }\end{array}$ & $\begin{array}{l}\text { En algunos de los casos } \\
\text { planteados es capaz de } \\
\text { realizar el despliegue de la } \\
\text { aplicación. }\end{array}$ & $\begin{array}{l}\text { No comprende el } \\
\text { Problema. En ninguno } \\
\text { de los casos planteados } \\
\text { es capaz de realizar el } \\
\text { despliegue de la } \\
\text { aplicación. } \\
\text { No intenta siquiera una } \\
\text { elaboración del } \\
\text { problema. }\end{array}$ \\
\hline \multicolumn{5}{|l|}{ CG13 Compromiso } \\
\hline $\begin{array}{l}\text { ER23.- } \\
\text { Comprometerse } \\
\text { éticamente }\end{array}$ & \begin{tabular}{|l|} 
En la totalidad de los casos \\
planteados, el estudiante \\
se compromete \\
éticamente e interactúa \\
con sus compañeros.
\end{tabular} & $\begin{array}{l}\text { En la mayoría de los casos } \\
\text { planteados, el estudiante } \\
\text { se compromete } \\
\text { éticamente e interactúa } \\
\text { con sus compañeros. }\end{array}$ & $\begin{array}{l}\text { En algunos casos } \\
\text { planteados, el estudiante se } \\
\text { compromete éticamente e } \\
\text { interactúa con sus } \\
\text { compañeros. }\end{array}$ & $\begin{array}{l}\text { No se compromete } \\
\text { éticamente ni } \\
\text { interactúa con sus } \\
\text { compañeros. }\end{array}$ \\
\hline \multicolumn{5}{|c|}{ CG14 Administración de proyectos } \\
\hline $\begin{array}{l}\text { ER22.- Ejecutar e } \\
\text { implementar de } \\
\text { forma práctica todas } \\
\text { las tareas que se } \\
\text { llevan a cabo en el } \\
\text { proceso de desarrollo } \\
\text { de software. }\end{array}$ & $\begin{array}{l}\text { Las tareas básicas que se } \\
\text { Ilevan a cabo en el proceso } \\
\text { de desarrollo de software } \\
\text { son ejecutadas e } \\
\text { implementadas en todas } \\
\text { las situaciones planteadas. }\end{array}$ & $\begin{array}{l}\text { Las tareas básicas que se } \\
\text { llevan a cabo en el proceso } \\
\text { de desarrollo de software } \\
\text { son ejecutadas e } \\
\text { implementadas en la } \\
\text { mayoría de las situaciones } \\
\text { planteadas. }\end{array}$ & \begin{tabular}{|l} 
Las tareas básicas que se \\
Ilevan a cabo en el proceso \\
de desarrollo de software no \\
son ejecutadas ni \\
implementadas en las \\
situaciones planteadas.
\end{tabular} & $\begin{array}{l}\text { No realiza la actividad. } \\
\text { No intenta la } \\
\text { elaboración del } \\
\text { problema. }\end{array}$ \\
\hline \multicolumn{5}{|l|}{ CG15 Implementación } \\
\hline $\begin{array}{l}\text { ER11.-Implementar } \\
\text { componentes de } \\
\text { software que utilicen } \\
\text { las técnicas } \\
\text { mencionadas. }\end{array}$ & $\begin{array}{l}\text { Todos los componentes de } \\
\text { software utilizan las } \\
\text { técnicas mencionadas en } \\
\text { clase. }\end{array}$ & $\begin{array}{l}\text { La mayoría de los } \\
\text { componentes de software } \\
\text { utilizan las técnicas } \\
\text { mencionadas en clase. }\end{array}$ & $\begin{array}{l}\text { Pocos componentes de } \\
\text { software utilizan las técnicas } \\
\text { mencionadas en clase. } \\
\text { No comprende el problema. }\end{array}$ & $\begin{array}{l}\text { No realiza la actividad. } \\
\text { No intenta la } \\
\text { elaboración del } \\
\text { problema. }\end{array}$ \\
\hline \multicolumn{5}{|l|}{ CG16 Divulgación } \\
\hline $\begin{array}{l}\text { ER24.- Desarrollar } \\
\text { capacidades de } \\
\text { divulgación frente a } \\
\text { un público } \\
\text { especializado y no } \\
\text { especializado. }\end{array}$ & $\begin{array}{l}\text { En todos los casos } \\
\text { planteados proporciona el } \\
\text { conocimiento exacto del } \\
\text { mensaje que transmite. Es } \\
\text { capaz de hacerlo } \\
\text { mediante palabras } \\
\text { habladas o por medio de } \\
\text { la representación gráfica } \\
\text { de signos y palabras } \\
\text { escritas. }\end{array}$ & $\begin{array}{l}\text { En la mayoría de los casos } \\
\text { proporciona el } \\
\text { conocimiento exacto del } \\
\text { mensaje que transmite. } \\
\text { Casi siempre es capaz de } \\
\text { hacerlo mediante } \\
\text { palabras habladas o por } \\
\text { medio de la } \\
\text { representación gráfica de } \\
\text { signos y palabras escritas. }\end{array}$ & $\begin{array}{l}\text { Sólo en algunos casos } \\
\text { proporciona el conocimiento } \\
\text { exacto del mensaje que } \\
\text { transmite. En pocos casos es } \\
\text { capaz de hacerlo mediante } \\
\text { palabras habladas o por } \\
\text { medio de la representación } \\
\text { gráfica de signos y palabras } \\
\text { escritas. }\end{array}$ & $\begin{array}{l}\text { No proporciona el } \\
\text { conocimiento exacto } \\
\text { del mensaje que } \\
\text { transmite. No es capaz } \\
\text { de hacerlo mediante } \\
\text { palabras habladas ni } \\
\text { por medio de la } \\
\text { representación gráfica } \\
\text { de signos y palabras } \\
\text { escritas. }\end{array}$ \\
\hline \multicolumn{5}{|l|}{ CG17 Diseño } \\
\hline $\begin{array}{l}\text { ER6.-Determinar los } \\
\text { requerimientos }\end{array}$ & $\begin{array}{l}\text { Determina en su totalidad } \\
\text { los requerimientos }\end{array}$ & $\begin{array}{l}\text { La determinación de los } \\
\text { requerimientos }\end{array}$ & $\begin{array}{l}\text { No determina } \\
\text { satisfactoriamente la }\end{array}$ & $\begin{array}{l}\text { No realiza la actividad. } \\
\text { No intenta la }\end{array}$ \\
\hline
\end{tabular}




\begin{tabular}{|c|c|c|c|c|}
\hline $\begin{array}{l}\text { funcionales y no } \\
\text { funcionales. }\end{array}$ & \begin{tabular}{|l|} 
funcionales y no \\
funcionales que se \\
desprenden del problema. \\
Provee información \\
adicional y una respuesta \\
completa.
\end{tabular} & $\begin{array}{l}\text { funcionales y no } \\
\text { funcionales es cercana a lo } \\
\text { esperado. Determina } \\
\text { dichos requerimientos en } \\
\text { la mayoría de los casos. }\end{array}$ & $\begin{array}{l}\text { mayoría de los } \\
\text { requerimientos funcionales } \\
\text { y no funcionales. No } \\
\text { comprende el problema } \\
\text { omitiendo partes } \\
\text { fundamentales de los } \\
\text { conceptos. }\end{array}$ & $\begin{array}{l}\text { elaboración del } \\
\text { problema. }\end{array}$ \\
\hline $\begin{array}{l}\text { ER8.- Diseñar módulos } \\
\text { con alta cohesión y } \\
\text { bajo acoplamiento }\end{array}$ & $\begin{array}{l}\text { Demuestra gran habilidad } \\
\text { en el diseño de módulos } \\
\text { en la totalidad de los } \\
\text { ejercicios propuestos. } \\
\text { Proponiendo además } \\
\text { buenos ejemplos e incluso } \\
\text { excediendo lo expuesto en } \\
\text { clase. }\end{array}$ & $\begin{array}{l}\text { Demuestra habilidad en el } \\
\text { diseño de módulos en la } \\
\text { mayoría de los ejercicios } \\
\text { propuestos. }\end{array}$ & $\begin{array}{l}\text { El diseño de los módulos es } \\
\text { pobre en la mayoría de los } \\
\text { ejercicios propuestos. No } \\
\text { demuestra conocimientos } \\
\text { sobre el tema. }\end{array}$ & $\begin{array}{l}\text { No realiza la actividad. } \\
\text { No intenta la } \\
\text { elaboración del } \\
\text { problema. }\end{array}$ \\
\hline
\end{tabular}

